\title{
Statistical Analysis of the Social Network and Discussion Threads in Slashdot
}

\author{
Vicenç Gómez \\ vgomez@iua.upf.edu
}

\author{
Andreas Kaltenbrunner \\ andreas.kaltenbrunner@upf.edu
}

Vicente López

vicente.lopez@barcelonamedia.org

\author{
Barcelona Media Centre d'Innovació, Barcelona, Spain \\ Dept. Tecnologia (DTIC), Universitat Pompeu Fabra, Barcelona, Spain
}

\begin{abstract}
We analyze the social network emerging from the user comment activity on the website Slashdot. The network presents common features of traditional social networks such as a giant component, small average path length and high clustering, but differs from them showing moderate reciprocity and neutral assortativity by degree. Using Kolmogorov-Smirnov statistical tests, we show that the degree distributions are better explained by log-normal instead of power-law distributions. We also study the structure of discussion threads using an intuitive radial tree representation. Threads show strong heterogeneity and self-similarity throughout the different nesting levels of a conversation. We use these results to propose a simple measure to evaluate the degree of controversy provoked by a post.
\end{abstract}

\section{Categories and Subject Descriptors}

J.4 [Computer Applications]: Social and Behavioral Sciences-Sociology; G.2.2 [Mathematics of Computing]: Graph Theory-Network problems, Trees

\section{General Terms}

Human Factors, Measurement, Performance

\section{Keywords}

social networks, online communities, bulletin board, weblogs, h-index, log-normal, power-law, thread, radial tree

\section{INTRODUCTION}

Message boards or web forums are online areas where discussions are held by many users on a variety of topics. Some users post articles and other users can comment on these posts, forming a discussion thread or nested dialogue. Although the first message boards, USENET and the bulletin board system (BBS), date back to 1979 only recently the social networks emerging from the comment interaction between their users have been studied [24, 14, 8].

In addition to this form of networks, message boards can show rich complexity in the structure of their discussion threads. Unlike personal weblogs which receive a few number of replies [15], message board blogs can receive thousands of messages during a day. Previous studies of USENET

Copyright is held by the International World Wide Web Conference Committee (IW3C2). Distribution of these papers is limited to classroom use, and personal use by others.

$W W W$ 2008, April 21-25, 2008, Beijing, China.

ACM 978-1-60558-085-2/08/04. have been focused mainly on visualization techniques to facilitate understanding of the social and semantic structure [20]. The Netscan system [22], a powerful interface to track discussion threads and authors, has proven to be a valuable tool to understand different roles appearing in these newsgroups $[6,4]$. It is therefore of interest to analyze the statistics governing the structure of threads in order to understand the underlying patterns of communication existing in these large online spaces, and to develop efficient techniques which improve the system performance.

Here we have selected Slashdot ${ }^{1}$, a popular technologynews website created in 1997 that publishes frequently short news posts and allows its readers to comment on them. Unlike other message boards, Slashdot has a community based moderation system that awards a score between -1 and +5 to every comment and upholds the quality of discussions by discouraging Spam and offensive comments [13]. Threads in Slashdot may trail for two weeks and one single post can easily exceed 200 contributions, which are mainly replies to other comments rather than direct responses to the original posts. These implicit relationships based on shared interests can be used to construct a network [24, 8$]$ amenable to study typical macroscopic quantities of complex networks [18].

In previous studies $[11,12]$ the temporal patterns of the time differences between a post and its comments have been analyzed and notable regularities were found. Here we use the same dataset as in these studies, which represents one year of activity on Slashdot and consists of about $10^{4}$ news posts which received 2, 075, 085 comments written by 93,636 users (see [12] for more details concerning the dataset).

We also characterize the discussion threads by studying several quantities associated to their radial tree structure. They show strong heterogeneity and self-similarity throughout the different nesting levels of a conversation. Based on these results, we propose a simple measure to evaluate the degree of controversy provoked by a post.

\section{THE SOCIAL NETWORK}

We first explain the procedure used to create three different versions of the network. Next, the values obtained for different indicators are analyzed with special emphasis on the degree distribution. Finally, we describe briefly the community structure.

\subsection{Building the Network}

We generate a social network based on the implicit relations between the author of a comment and the user who

\footnotetext{
${ }^{1}$ http://www.slashdot.org
} 


\begin{tabular}{|c|c|r||c|c|}
\hline filter & total cmnts & \multicolumn{1}{|c|}{$\%$} & step cmnts & \multicolumn{1}{c|}{$\%$} \\
\hline Post & 473,065 & 22.8 & 473,065 & 22.8 \\
Anonymous & 385,901 & 18.6 & 295,396 & 14.2 \\
Low score & 45,785 & 2.2 & 9,691 & 0.4 \\
Self-replies & 56,489 & 2.7 & 15,045 & 0.7 \\
\hline
\end{tabular}

Table 1: Comments discarded after proper filtering.

replies to it. To improve the quality and the representativity of the resulting graph, we filter some of the comments according to the following four criteria:

1. The post: Under this assumption, no relations exist between the post's author and its direct commentators, unless he also participates later in the discussion.

2. Anonymous comments were also discarded.

3 . We discard very low quality comments with score -1 .

4. Finally, we filter out self-replies, often motivated by a forgotten aspect or error fix of the original comment.

The second and third column of Table 1 show the total number and the percentage of comments which fall in each category. Columns 4 and 5 give number and percentage of comments discarded due to the above explained filter steps. Note that after elimination of direct replies to posts and anonymous comments, low-score comments only represent a small fraction.

After the filtering process, the remaining number of comments is $1,281,888$, approximately $63 \%$ of the total. The users are reduced to 80,962 , approximately $87 \%$ of the initial set of users.

In a social network, each user corresponds a node $i \in V$ in a graph $G=\langle V, E\rangle$. Edges in our graph $(i, j) \in E$ indicate social relations between two individuals, which are consequence of their comment activity. Let $n_{i j}$ be the number of times that user $i$ writes a comment to user $j$. Based on $n_{i j}$, there are several ways to interpret when two users are linked. To be as much systematic as possible, we compare three different types of networks according to the following interpretations:

Undirected dense : An undirected edge exists between users $i$ and $j$ if either $n_{i j}>0$ or $n_{j i}>0$. The weight of that edge $w_{i j}$ is simply the sum $n_{i j}+n_{j i}$.

Undirected sparse : An undirected edge exists between users $i$ and $j$ if $n_{i j}>0$ and $n_{j i}>0$. The weight of an edge $w_{i j}$ in this case is defined as $w_{i j}=\min \left\{n_{i j}, n_{j i}\right\}$.

Directed : A directed edge exists from user $i$ to user $j$ if $n_{i j}>0$ regardless of $n_{j i}$. The weight $w_{i j}=n_{i j}$.

Figure 1 shows a small example to illustrate the generation of the three different graphs. On the left, there is a tree structure corresponding to a small thread of depth 4 . Labels denote the user who writes the contribution and valid comments are shown within the gray region. The post triggers four responses from users A, B, C and D. At the second nesting level, five comments appear (two from the same user $\mathrm{E}$, one from user A (who already commented on the original post), and two more from users $F$ and G. At the third level, there are only two comments from users $\mathrm{A}$ and $\mathrm{C}$, and finally, there is one last comment from G.
The small graphs on the right correspond to the three graph versions. In Figure $1 \mathrm{~b}$, users are linked if they exchange at least one message. In Figure 1c, bidirectional edges exists between users when both users replied a comment of the other. These reciprocal relations are the links in the undirected sparse graph. Note that we do not consider possible relations not associated to the thread structure like mentioning a user within the text of a comment. Semantic analysis would be required to overcome this limitation.

\subsection{General Description}

We now characterize the structural properties of the obtained graphs [18]. Table 2 shows the values of the indicators considered here for the different networks. If not stated otherwise, indicators are calculated for the unweighted graph.

In the first two rows we show the number of nodes $N=|V|$ and edges $M=|E|$ of the respective networks. In the case of the undirected sparse graph, $N$ is reduced significantly. The number of actual links $M$ is very small compared to the potential number of relationships $\mathcal{O}\left(N^{2}\right)$. This would suggest a highly sparse network with many connected components composed of small groups of users. However, as row 3 of Table 2 indicates, a vast majority of the population forms a "giant component", leaving only a small proportion of users disconnected from that component. These isolated users are grouped mainly in pairs, or at most, in small clusters of size 4 in all three networks. In both undirected graphs, the "giant component" contains more than $97 \%$ of the users and in the directed ${ }^{2}$ network almost $75 \%$. These quantities indicate that the social network of Slashdot is characterized by a compact community and a small proportion of isolated users, in concordance with typical social networks.

The average degree $\langle k\rangle$ is shown in row 4 of Table 2 (standard deviations between parenthesis). The directed network presents an intermediate value between the dense and sparse undirected representations. All cases show high standard deviations, indicating a big level of heterogeneity within the community. This aspect is analyzed in more detail in Section 2.3 .

The average path length $\ell$, measured only for the giant component, takes small values for all three networks, sug-

\begin{abstract}
${ }^{2}$ We consider weakly connected components in the directed case, i.e. two vertices $i$ and $j$ belong to the same component if there exists a path between $i$ and $j$ at least in one of the two possible directions. The size of the big cluster for strongly connected components is of course, smaller.
\end{abstract}

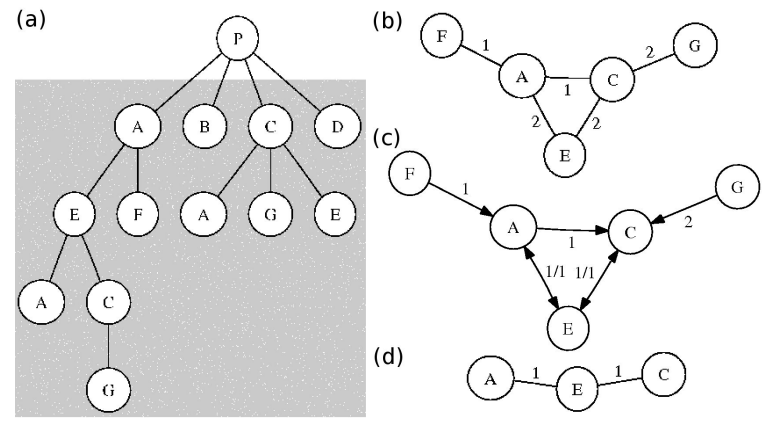

Figure 1: Example of graph generation. (a) A small thread of comments. (b) Undirected dense network. (c) Directed network. (d) Undir. sparse network. 


\begin{tabular}{|c|c|c|c|}
\hline Indicator & Directed & Und.Dense & Und.Sparse \\
\hline$N$ & 80,962 & 80,962 & 37,087 \\
$M$ & $1,052,395$ & 905,003 & 294,784 \\
Max.clust. & $73.12 \%$ & $97.90 \%$ & $97.15 \%$ \\
$\langle k\rangle$ & $13(50.1 / 49.4)$ & $22.36(79.3)$ & $7.95(25.7)$ \\
\hline$\ell$ & $3.62(0.7)$ & $3.48(0.7)$ & $4.02(0.8)$ \\
$\ell_{\text {rand }}$ & 4.38 & 3.62 & 5.05 \\
\hline$D$ & 10 & 9 & 11 \\
\hline$C$ & $0.027(0.075)$ & $0.046(0.12)$ & $0.017(0.078)$ \\
$C^{w}$ & $0.026(0.074)$ & $0.047(0.12)$ & $0.018(0.080)$ \\
$C_{\text {rand }}$ & $1.67 \cdot 10^{-4}$ & $2.88 \cdot 10^{-4}$ & $2.27 \cdot 10^{-4}$ \\
\hline$r$ & -0.016 & -0.039 & -0.016 \\
$\rho$ & 0.28 & - & - \\
\hline \multicolumn{4}{|c}{} \\
\hline
\end{tabular}

Table 2: Indicators of the Slashdot social networks.

gesting that the small-world property is present in all of them. The quantities are approximately one unit lower than the corresponding value for a random graph $\ell_{\text {rand }}$. The maximal distance $D$ between two users is also very small. Even for the undirected sparse case, it only takes a maximum of eleven steps to reach a user starting randomly from any other. These results are also in accordance with similar studies of other traditional social networks.

To study the statistical level of cohesiveness we calculate the clustering coefficient $C$ according to [23], and also its weighted version $C^{w}[1]$. We notice no significant differences between them. Thus the number of messages interchanged between two users is not relevant to determine the clustering level. The impact of having a weighted network is analyzed in more detail in Section 2.5. We can see that for all graphs, $C$ and $C^{w}$ are about two orders of magnitude higher than their randomized counterpart $C_{\text {rand }}$. This is again in harmony with other analysis of real-world networks, which report similar deviations from the random graph, and enhances the evidence of the small-world property. As before, the directed graph represents an intermediate value between both undirected versions.

Another quantity of special interest in social networks is the degree correlation, or mixing coefficient, which allows to detect whether highly connected users are preferentially linked to other highly connected ones or not. This fact is known as assortative mixing by degree and is present in many social networks [19]. Table 2 shows the correlation coefficient $r$ for our three networks, which is far from \pm 1 . Therefore, unlike traditional social networks which present a strong assortative mixing, Slashdot is characterized by neither assortative nor dissortative mixing. Users do not show any preference to write comments in function of the connectivity of the other users. Interestingly, other related studies of online communities show similar $[10,8]$ coefficients. This seems to be a fundamental difference to social interactions occurring outside these large online spaces.

The last general property we analyze is the reciprocity. High reciprocity is another feature typically present in social networks. In our case, reciprocity occurs when a user $i$ replies the answer of another user $j$ to a previous comment of $i$, and can be measured by means of a reciprocity coefficient $\rho$. Using the method proposed in [7], we quantify how the Slashdot network differs from a random network in the presence of two mutual links (edges in both directions) between pairs of nodes. The small positive value $\rho=0.28$ suggests that our network is only moderately reciprocal, so that users tend to write slightly more often than expected by chance to other users who previously wrote them.

From this global characterization we can conclude that the underlying network of Slashdot presents common features of traditional social networks, namely, a giant cluster of connected users, small average path length and high clustering. In contrast to other social networks, Slashdot shows moderate reciprocity and neutral assortativity by degree. We also see that there is significant difference between considering dense and sparse undirected versions, and that the directed version represents an intermediate description between the two. The moderate value of the reciprocity coefficient $\rho$ suggests that studying only the undirected network, one could miss some relevant structural information. Finally, regarding clustering, we see no significant differences between the weighted and the binary network. Despite the strong similarity to a much smaller network of BBS-users [8], these two features seem to be exclusive of Slashdot.

\subsection{Degree Distributions}

We now focus on the function describing the number of users in the network with a given number of neighbors. The analysis of this degree distribution describes the level of interaction between users and provides a robust indicator about the grade of heterogeneity in the network.

Figure 2 shows in small circles the probability distribution (pdf) and the cumulative distribution (cdf) of the degrees for the directed network. The other two networks present equivalent results. First, we can see that in and out degree distributions are almost identical. Unlike previous studies [8], in our case the activities of writing and being replied could be characterized by similar processes.

As expected, the obtained distributions are heavy-tailed, covering in this case more than three orders of magnitude and indicating a high level of heterogeneity between the users. Surprisingly, the users located at the tail of the distri-
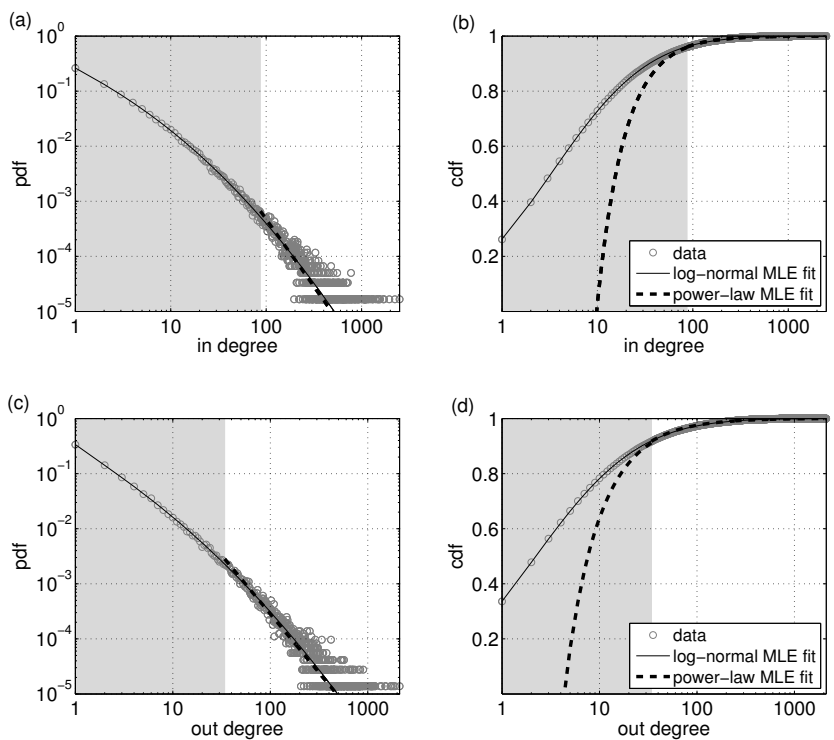

Figure 2: In and out degree distributions of the directed Slashdot network and corresponding PL and truncated LN approximations. 
bution are not Slashdot authors of posts who also participate actively in the discussions, as one would expect. The first Slashdot author in the list sorted by degree appears at position number 378 (in the case of the in-degree distribution of the directed network). Therefore, the hubs of the social network are not the "affiliated" authors, but regular users who participate actively in the discussions.

To find a functional form which best explains the observed data, we compare two approximations: the "usually assumed" power-law (PL) hypothesis, and a truncated lognormal (LN) hypothesis [16]. Their corresponding density functions are given by

$$
\begin{gathered}
f_{L N}(x ; \mu, \sigma, \theta)=\frac{1}{(x-\theta) \sigma \sqrt{2 \pi}} \exp \left(\frac{-(\ln (x-\theta)-\mu)^{2}}{2 \sigma^{2}}\right) \\
\text { and } f_{P L}\left(x ; \alpha, x_{\text {min }}\right)=\frac{x^{-\alpha}}{\zeta\left(\alpha, x_{\text {min }}\right)},
\end{gathered}
$$

where $\zeta(\alpha, x)=\sum_{n=0}^{\infty}(n+x)^{-\alpha}$ is the generalized or Hurwitz zeta function. We select the optimal parameter values using maximum likelihood estimation (MLE). The PL distribution has as parameters the scaling exponent $\alpha$ and the minimum degree value $x_{\text {min }}$ from which the PL behavior occurs. To find the proper value of $x_{\min }$ we apply a recent method proposed in [5]. The LN distribution has three parameters: the mean $\mu$, the standard deviation $\sigma$ and a shift $\theta$, which represents a lower bound of the degree values. Both distributions can be very similar [16].

Figure 2 shows that for the case of the PL hypothesis (dashed-bold line), the obtained values of $x_{\min }$ (represented by the border between gray and white areas) are extremely large, leaving almost all the data samples outside the fitted region, which contains only a few users at the tail of the distribution. However, the LN fit provides an explanation of the entire dataset, of both, the left-support of the distribution, where most of the probability mass is concentrated, and also in the tail of the distribution, where fluctuations are bigger due to finite sampling effects.

Table 3 summarizes the results of the fits of the degree distributions. The first two columns of the upper part of Table 3 show the parameters of the PL distribution. The minimum value of $x_{\min }$ is 5 , corresponding to the undirected sparse version. Even in this case, more than $75 \%$ of the users are not included in the PL fit. The proportion of discarded samples is indicated in the third column. The lower part of Table 3 gives the parameters of the LN approximation, which show more variability than those of the PL.

\begin{tabular}{|c||c|c|c|c|}
\hline \multicolumn{5}{|c|}{ power-law } \\
\hline Network type & $x_{\min }$ & $\alpha$ & $\%$ disc. & p-value \\
\hline undir. dense & 85 & 2.27 & 94.49 & 0 \\
dir. in-degree & 87 & 2.44 & 96.05 & 0 \\
dir. out-degree & 34 & 2.13 & 91.68 & 0 \\
undir. sparse & 5 & 1.92 & 75.85 & 0 \\
\hline \hline \multicolumn{5}{|c|}{ truncated log-normal } \\
\hline Network type & $\mu$ & $\sigma$ & $\theta$ & p-value \\
\hline undir. dense & 1.03 & 2.04 & 0.32 & $\mathbf{0 . 4 3}$ \\
dir. in-degree & 1.14 & 1.87 & 0.47 & $\mathbf{0 . 9 3}$ \\
dir. out-degree & 0.45 & 2.07 & 0.39 & $\mathbf{0 . 5 8}$ \\
undir. sparse & -0.73 & 2.14 & 0.19 & $\mathbf{0 . 9 9}$ \\
\hline
\end{tabular}

Table 3: PL and LN fit of degree distribution.
After selecting the optimal values of the parameters for both hypothesis, we test whether the provided model of the data can be accepted or not. We use the KolmogorovSmirnov test (KS), whose p-values are shown in the last column of Table 3. In all cases, the PL hypothesis provides a p-value much lower than 0.1 (our choice of the significance level of the KS-test). Hence, we can conclude that, even after discarding most of the data, the PL is not able to explain the tail of the distributions. In contrast, the obtained p-values for the truncated LN model are quite high, all of them bigger than 0.1 , so the LN-hypothesis allows to explain the entire distribution.

\subsection{Mixing by Score}

In Section 2.2 we have seen that the Slashdot network presents neutral mixing by degree. It is interesting to analyze whether Slashdot users show assortative mixing by other attributes. In this subsection, we associate to each user a score, which is calculated by averaging over all the scores of the comments of the same user. This quantity allows us to differentiate high-quality writers (those with high mean score) from regular-quality writers.

The initial score of a comment is generally 1 if it comes from a registered user or 0 if it is anonymous ${ }^{3}$. Moderation can modify the initial score to any integer within the range $[-1,5]$. To ensure a representative subset of the network, we only consider users who wrote at least 10 comments, a total of 18, 476 users, representing approximately $23 \%$.

In Figure 3a we plot the histogram and the corresponding cdf of the distribution of the mean scores. Note that the minimum score is 0 , since we eliminate -1 comments. The distribution shows an unexpected bimodal profile, with two peaks at mean scores 1.1 and 2.3. This indicates that two different classes of users coexist.

Is the mean score a representative measure of the user's commenting quality? To check its validity we plot in Figure $3 \mathrm{~b}$ the distribution of the standard deviations of the scores. More than 3/4 of all users show deviations smaller than 1 , so the scores a user obtains do not fluctuate significantly. Therefore, their mean seems to be a good candidate to characterize the user.

${ }^{3}$ Anonymous users cannot be considered in this analysis.
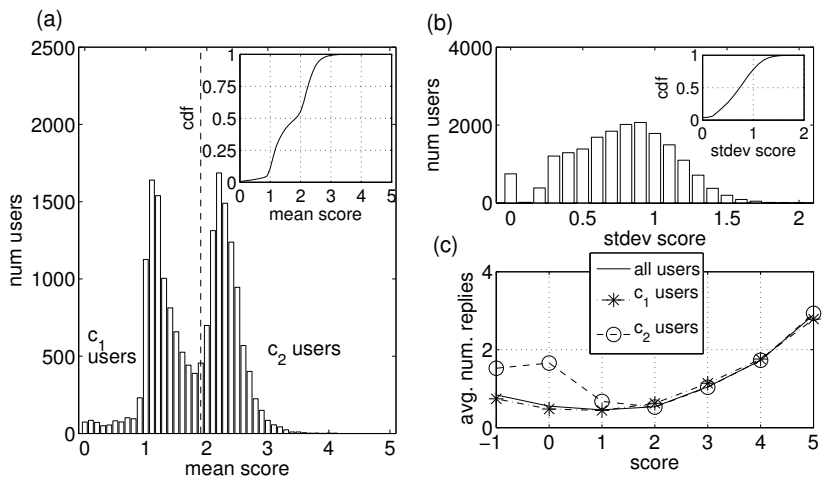

Figure 3: (a) Mean scores of users who wrote at least 10 comments. (b) Standard deviations of the mean scores of the same users. (c) Relation between the score of a comment and the average number of received replies for all, $c_{1}$ and $c_{2}$ users. 
We now analyze whether users of one class reply preferentially to users of the same class or not. The bimodality suggests a simple characterization using two classes of users. We select the boundary between classes to balance their sizes (the resulting boundary is 1.90). Thus a given user is assigned to class $c_{1}$ if its mean score is $\leq 1.90$, and otherwise to class $c_{2}$. Class $c_{1}$ contains 9,254 users whereas $c_{2}$ contains 9,222 . The assortativity matrix $\mathbf{E}$ is calculated counting the number of comments interchanged between classes. Each element $E_{i j}$ indicates the number of comments that users of class $i$ wrote to users of class $j$. Its normalized version $\mathbf{E}^{\prime}$ is obtained dividing $\mathbf{E}$ by the total number of comments:

$$
\mathbf{E}=\left(\begin{array}{rr}
78,341 & 198,391 \\
151,013 & 455,997
\end{array}\right) \quad \mathbf{E}^{\prime}=\left(\begin{array}{ll}
0.09 & 0.22 \\
0.17 & 0.52
\end{array}\right) .
$$

The assortativity coefficient is $r_{\text {score }}=0.036$, so the network is neutrally mixed by mean score. Note, however, that more than half of the comments are written from users of class $c_{2}$ to other $c_{2}$ users, and that the proportion of comments received by $c_{2}$ users is 0.74 , so there is a strong bias in favour of good writers.

This can either mean that users tend to reply preferentially to users of the class with higher average score, or simply that high-scored comments tend to receive more reactions than low-scored ones independently of the user. To check this, we compare in Figure 3c the average number of replies received by comments in function of their scores for either all users or only the users of classes $c_{1}$ and $c_{2}$. To get a broader range of scores, we also include negative scores.

It is quite clear that scores $\geq 2$ correlate with the average number of reactions and are independent of the user's class, but comments with scores below 2 do not show this correlation and achieve substantially more replies on average if written by users of class $c_{2}$.

We can thus conclude that on average, although higher scored comments tend to achieve more replies regardless of the user who wrote it, it is also true that good writers, even when they post low-scored comments, still receive significant more replies than $c_{1}$ users.

\subsection{Community Structure}

To end this characterization of the Slashdot network we analyze its community structure. We take a simple approach based on agglomerative clustering which takes benefit from the weighted nature of the Slashdot network [18]. We choose the dense undirected network and start our procedure with each node as an independent cluster. Let $\lambda$ denote the number of comments, so that pairs of users $(i, j)$ who interchange a number of comments $w_{i j} \geq \lambda$ are included in the network, and the other connections are discarded. Starting from the biggest value $\lambda=\lambda_{\max }$ and progressively decreasing it, users are connected incrementally and communities can be obtained. This simple procedure is equivalent to building a dendrogram and allows to browse through the community structure at different scales by changing the parameter $\lambda$.

Figure 4 a shows the distribution of the weights $w_{i j}$ of all links in the network. The vast majority of pairs of users only exchanges a small number of comments whereas a few of them really maintain intense dialogues during the year. This seems to be the reason why previous properties such as the clustering coefficient do not show significant differences between the weighted and the unweighted network. The most discussing pair of users exchanged a total of 108
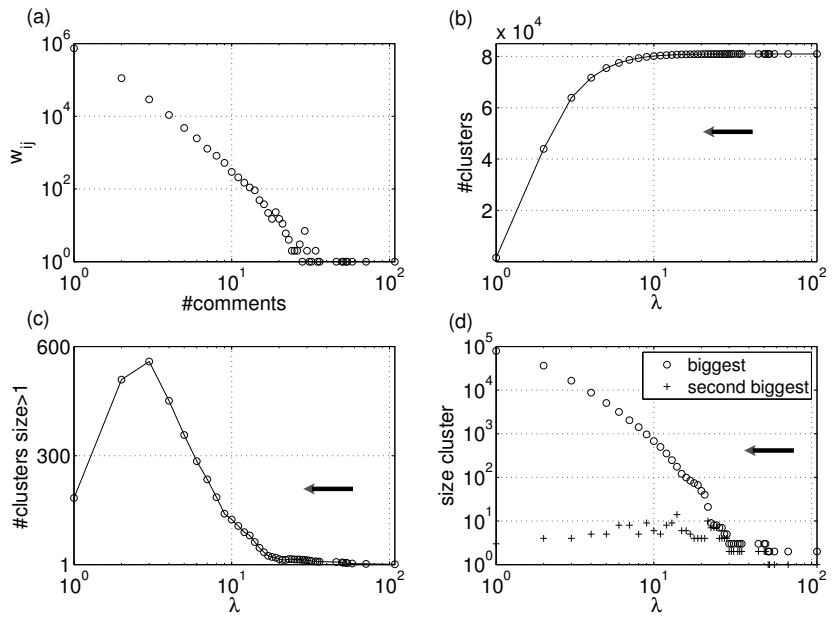

Figure 4: Results of the agglomerative clustering. (a) Distribution of weights (number of messages between pairs of users). (b) Number of clusters in function of $\lambda$ and (c) considering only clusters of size $>1$. (D) Size of the two biggest clusters.

comments. This represents our initial value $\lambda_{\max }$ to start the agglomerative procedure.

Since most of users exchange only a small amount of comments, one would expect that the number of clusters remains quite high for a wide range of $\lambda$ values. This is indeed the case. As $\lambda$ is progressively decreased, users are being grouped in small clusters. Simultaneously, a giant cluster is being formed which absorbs the small clusters when they reach a moderate size. In Figure $4 \mathrm{~b}$ we plot the number of clusters in function of $\lambda$. It is reduced dramatically in the last step when $\lambda=1$ is reached. A more detailed analysis can be obtained if we discard isolated users, and focus only on groups of pairs or more users, who at least interchanged one comment. This is shown in Figure 4c. We can see that for high values of $\lambda$, the number of groups of size two or more is very small. Then it starts to grow significantly around $\lambda \sim 10$, reaches a maximum at $\lambda=3$, and then again falls to the number of components of the original graph (considering all links). We also plot in Figure $4 \mathrm{~d}$ the sizes of the two biggest components in function of $\lambda$. We can see that the biggest component grows very fast and the second biggest remains small, showing evidence of a giant cluster present in all scales.

We can track the resulting communities and show the networks at each $\lambda$. This is roughly illustrated in Figure 5, where two snapshots of the agglomerative process are shown. Figure 5 (top) corresponds to a high value of $\lambda=20$, where a small backbone of the most connected users is starting to grow. Note that users are colored according to their score attribute (see Section 2.4). Users corresponding to the second class (high-quality commentators) are colored in red. Clearly, the backbone of Slashdot users is formed mainly by high-quality commentators. For $\lambda=15$ (Figure 5 bottom), the most connected users receive even more connections and form the giant component. A few clusters of small sizes which still do not have grown sufficiently to be merged with the big community are also present. 


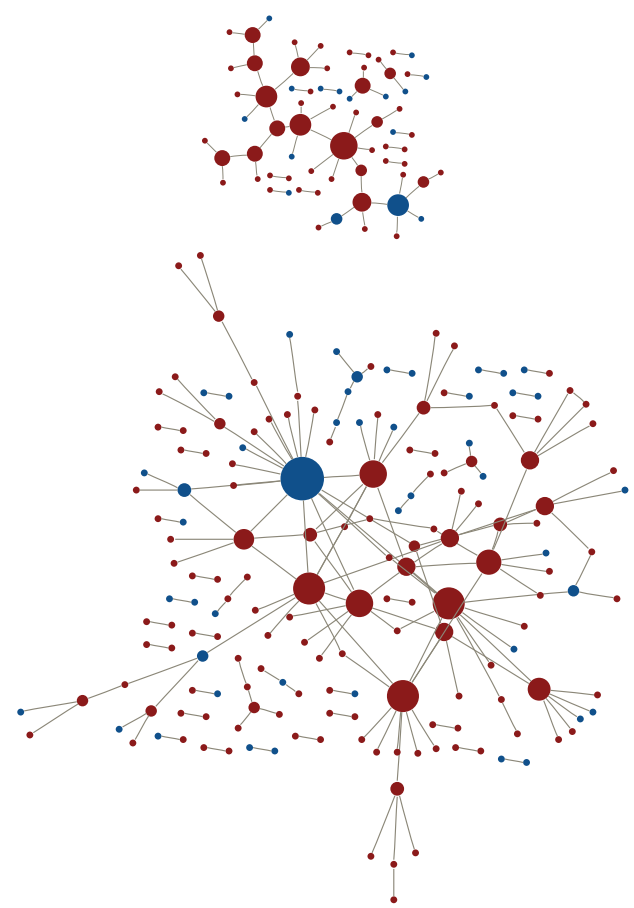

Figure 5: Two snapshots of the network for $\lambda=20,15$. Nodes are colored according to their score class (red: high quality, blue: medium quality). For clarity, we only show clusters of size $>1$.
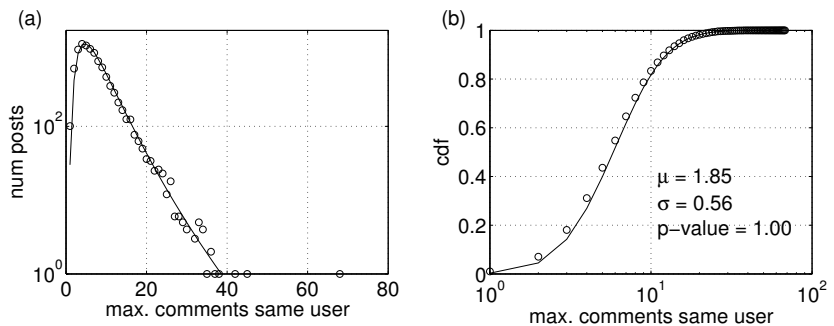

Figure 6: (a) Histogram of the maximum number of comments of one single user per post. Solid line corresponds to the best $\mathrm{LN}$ fit (b) cdf.

\section{STRUCTURE OF THE DISCUSSIONS}

After analyzing the social network of Slashdot, we focus on the question about how information is structured within a discussion thread. A thread starts with the publication of a post, which in turn triggers an amount of activity in the form of comments. In this section, we present a statistical characterization of the structure of such discussions using a useful and intuitive radial tree representation. This representation leads naturally to a measure which can be useful to evaluate the degree of controversy of a given post.

An initial picture of the activity generated by posts can be found in previous studies [12]. Posts receive on average approximately 195 comments and there exists a clear scale in the number of comments a post can originate. Half of them receive less than 160 contributions. A small number of highly discussed ones, however, can trigger more than one thousand contributions.
The number of comments gives an idea of how the participation is distributed among the different articles, but is not enough to quantify the degree of interaction. For instance, a post may incite many readers to comment, but if the author of a comment does not reply the responses to his comment, there is no reciprocal communication within the thread. In this case, although users can participate significantly, we can hardly interpret that the post has been highly discussed. On the other hand, a post with a small number of contributors but with one long dialogue chain will evidence a high degree of reciprocal interaction (albeit its general interest may be reduced). At the description level of the social network, the reciprocity coefficient $\rho$ and the agglomerative clustering described in the previous section already represent a measure to explain the degree of (reciprocal) interaction. At the description level of the individual post, a possible measure to quantify this type of interaction is the maximum number of comments written by a single user to a particular post.

We show this quantity (excluding the anonymous users) versus the number of posts, i.e. how many posts exist with a certain maximum number of comments written by the same user, in Figure 6. The obtained distribution has a peak at 4. As the cdf indicates, for approximately half of the posts at least one single user participates 5 times or more in the discussion. The log-normal shape of the distribution suggests a multiplicative process underlying the generation of this quantity. This indicates a strong heterogeneity and level of interaction within discussion threads. Users do not only give an opinion, but also interchange a significant quantity of messages, and the intensity of this interaction varies considerably throughout the different posts. We now study in more detail their intrinsic tree structure.

\subsection{Radial Tree Representation}

The high number of comments elicited by controversial posts makes them difficult to explore and to find relevant contributions within the nested dialogues. The current interface of Slashdot offers a filtering mechanism based on scores. By default, direct comments to a post rated 1 or higher are fully shown. For deeper nesting levels, comments can be fully shown (score 4 or above), abbreviated (score between 1 and 4 ) or hidden (score below 1 ).

We propose a natural representation of thread discussions which takes advantage of their structure. Consider a post as a central node. Direct replies to this post are attached in a first nesting level and subsequent comments at increasing nesting levels in a way that the whole thread can be considered as a circular structure which grows radially from a central root during its lifetime, a radial tree. Figure 7 shows three snapshots of a radial tree associated to a controversial post which attracted a lot of users. An analog example of a less discussed post can be seen in Figure 8. More examples of trees are shown in Figure 9. Their profiles are highly heterogeneous. In some examples, only a huge number of contributions without replies appear in the first level, resulting in trees with high widths but small depths. In other examples, however, there are only discussions between two users who comment alternatively giving rise to very deep trees with small widths. Sometimes, the intensity of the discussion is translated to one of the branches because of a controversial comment which triggers even more reactions than the original post (e.g the post in the center of Figure 9). 


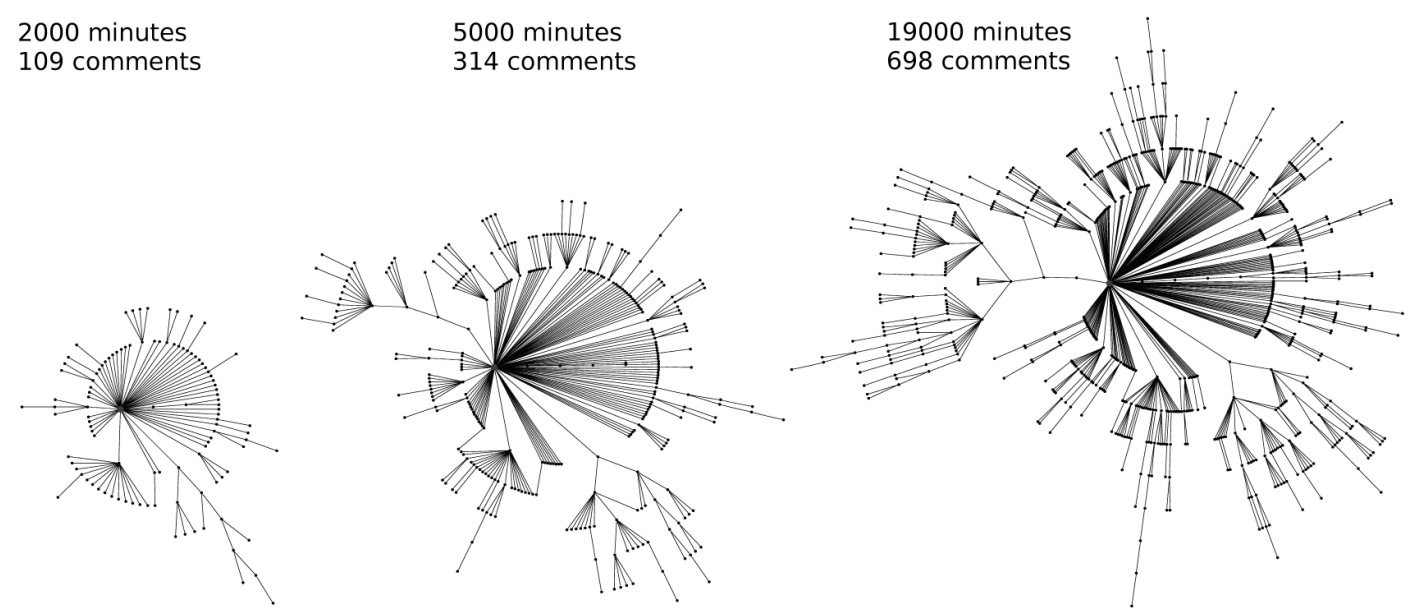

Figure 7: An example of radial tree structure corresponding to a controversial post related to Windows and Linux which received a total of 982 comments. The title of the post is "Can Ordinary PC Users Ditch Windows for Linux?". Figures show three snapshots in different times.

Apart from being a useful tool for browsing and examining the contents of a highly discussed post, radial trees can be used to describe statistically how information is structured in a thread. In Figure 10a we plot the distribution of all the extracted comments per nesting level for all posts. This gives an idea about the relation between the width versus the depth of the trees. The first two levels contain most of the comments and then their number decays exponentially in function of the depth. The maximum depth was 17. A general pattern which seems to be common to all threads is formed by a broad first nesting level of contributions, followed by a second, even wider set, and finally an exponential decay. This fact is reflected in the peak at depth two of the plot and the decreasing number of comments for deeper nesting levels. This gives evidence of the transient nature of the discussions. The reason behind this pattern is apparently related with an initial growth of interest which is reduced after users may have exposed all their knowledge, or translated to a more recent article. Only those who have engaged a dialogue will keep writing in subsequent levels. This decay could also be explained because of accessibility awkwardness, since the visibility of a comment can be proportional to its depth. The previous result would suggest that the majority of posts does not reach high nesting levels, and one would expect a similar distribution for the

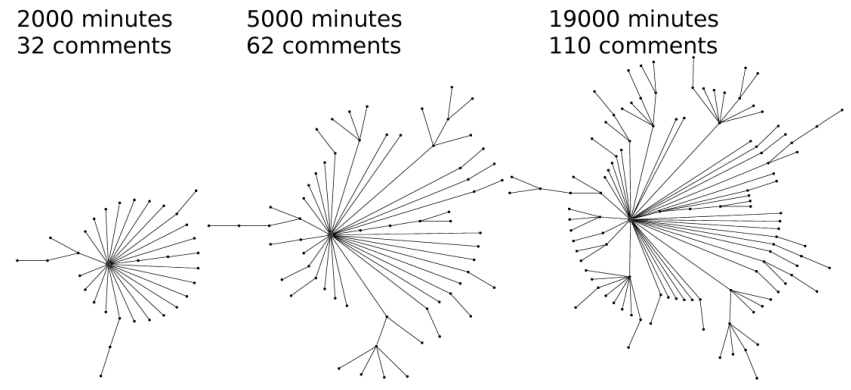

Figure 8: Radial tree structure of a little commented post which received 133 comments in total. Its title is "Amazon One-Click Patent to be Re-Examined". maximum depth of the posts. However, as Figure 10b indicates, the distribution of maximum depths does not follow the same pattern. It is almost symmetrical, weakly skewed toward smaller maximum depths. Although comments are concentrated in the first levels, threads typically reach a depth around 7 .

Up to now, the quantities analyzed do not capture the apparent heterogeneity of the discussion threads we have reported in previous examples (see Figure 9). We now take
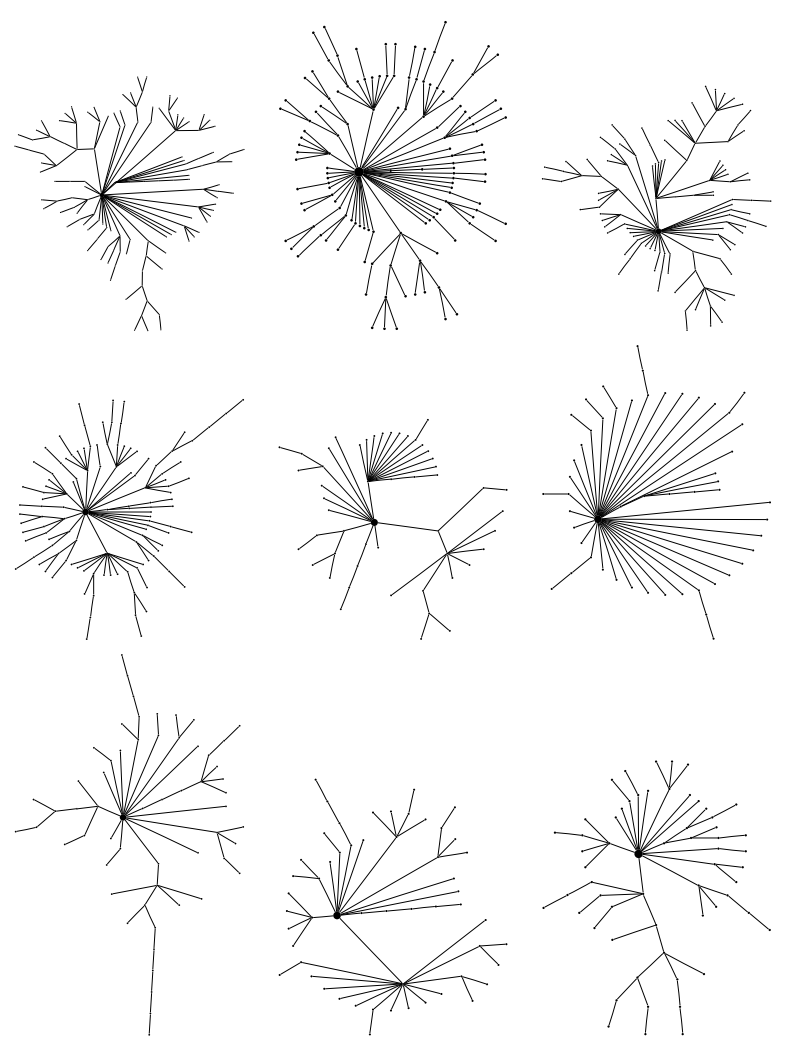

Figure 9: Heterogeneity in the radial trees. 

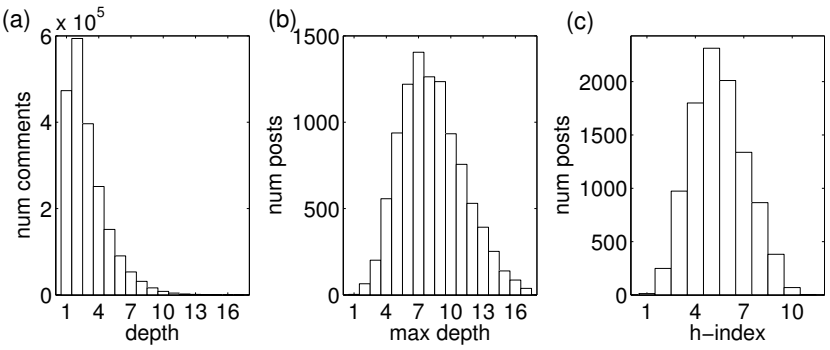

Figure 10: Results of (a) Number of comments per nesting level. (b) Number of posts per maximum depth. (c) Number of posts per h-index.

a look on how the comments are generated within a given nesting level. This analysis can be performed extracting the branching factors $b$, that is, the number replies provoked by a given comment (or a given post). Figure 11 shows in log-log scale the distributions of $b$ for the first five nesting levels. Level 0 corresponds to direct comments to the posts, whereas subsequent plots correspond to replies to comments. First, the range of possible values spans almost three orders of magnitude for direct comments and is considerable for subsequent nesting levels, which gives evidence of the high heterogeneity underlying the discussion threads. Second, there is a clear discrepancy between commenting level 0 and subsequent ones. While the distribution of direct replies follows quite closely a bell-shape in the log-log domain, subsequent levels have an always decreasing probability. This illustrates the different nature of the process underlying the generation of comments to the initial post and the generation of replies to other comments. Interestingly, this variation is not reported in subsequent nesting levels. In addition, no dependency of the score on the nesting level could be found (data not shown). Although the resulting threads can take very different forms as we have previously shown, the same generative process seems to be taking place at all nesting levels. The bell-shaped curve of the first level branching factors, and the curvature in subsequent levels in log-log scales suggest again a good LN fit to
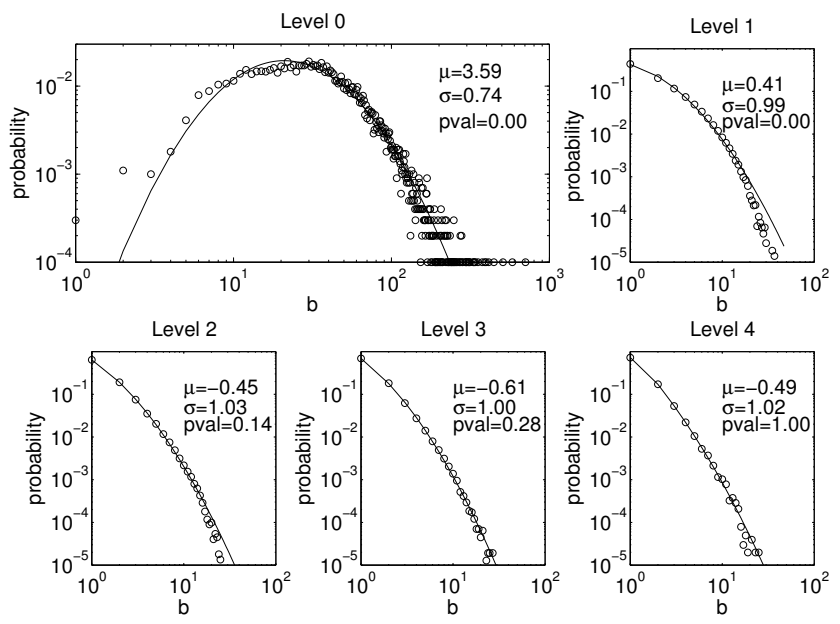

Figure 11: Distributions of branching factors $b$ in 5 levels. Level 0 shows direct comments to posts.

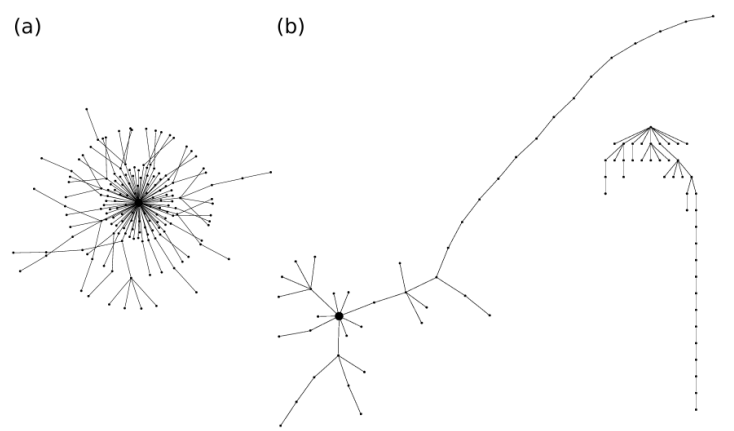

Figure 12: (a) Thread with many comments in the first level, but a few in subsequent levels. (b) Thread with a intense debate between two users.

explain the observed data, indicated by continuous lines in Figure 11. However, according to the p-values of KS-tests, the LN hypothesis is only accepted for levels deeper than 2.

\subsection{The $\mathbf{H}$-index as a Structural Measure of Controversy}

In this subsection we will use the previous results to measure the degree of controversy of a post. As before, our approach does not consider semantic features and only relies on its structural information. It is important to note that a definition of controversial is necessarily subjective. However, indicators such as the number of comments received or the maximum depth of the discussions can be, among others, good candidate quantities to evaluate the controversy of a post, but suffer from some drawbacks as we will explain in what follows. We therefore seek for a measure, as simple as possible which incorporates as many of these factors and is able to rank a set of posts properly. The number of comments alone does not tell us much about the structure of the discussion. There might be a lot of comments in the first level but very little real discussions, such as in the post of Figure 12a. A better measure for the controversy of a post seems to be the maximum depth of the nesting. But again that measure has some drawbacks. Two users may become entangled in some discussion without participation of the rest of the community, increasing the depth of the thread. The example of Figure $12 \mathrm{~b}$ illustrates this case. We thus want to overcome both types of bias.

We propose to quantify the degree of controversy associated to a post using an adapted version of the h-index [9], commonly used to characterize the scientific output of researchers. The papers of a researcher are ordered by their number of citations in descending order and the h-index is then defined as the maximum rank-number, for which the number of citations is greater or equal to the rank number. It represents a fair quantity which considers the number of papers published by the scientist and their visibility, or how often these papers are cited by other scientists. Some extensions of this index have been proposed as an alternative to the impact-factor of journals and conferences [3, 21]. See [2] for more details and a review on literature about the h-index.

For our purposes, we will define the h-index in the following way: given a radial tree corresponding to a discussion thread and its comments organized in nesting levels, the hindex $h$ of a post is then the maximum nesting level $i$ which has at least $h>i$ comments, or in other words, $h+1$ is the 


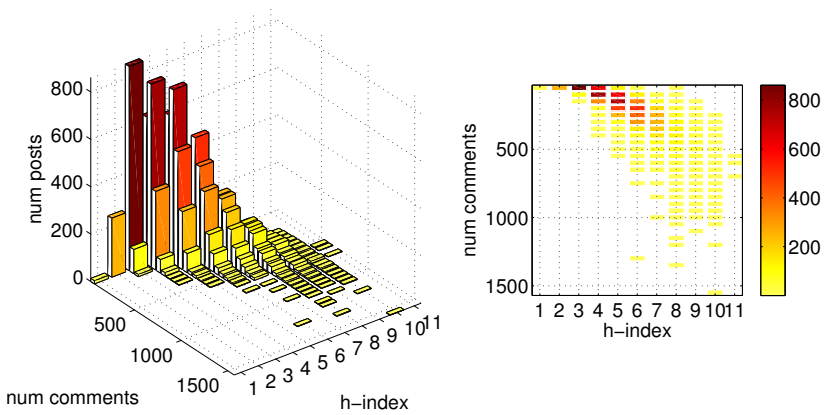

Figure 13: h-index versus number of comments.
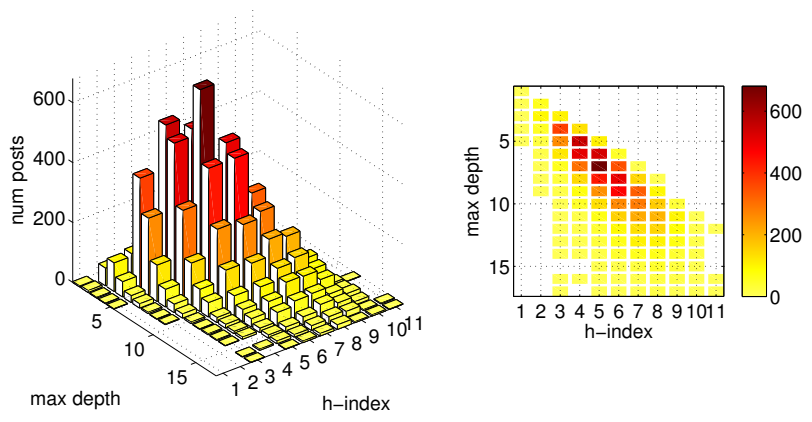

Figure 14: h-index versus maximum depth.

first nesting level $i$ which has less than $i$ comments. Turning back to Figure 12b, we can easily calculate the h-index. There are 9 comments in both first and second levels, 6 comments in the third level and 3 comments in the fourth level, which gives an h-index of 3 . This post has maximum nesting level of 17 , and it is ranked first if only the maximum depth is considered, but drops down to the position 9, 239 using the h-index. Similarly, the post of Figure 12a, which received 161 comments, has just an h-index of 3 , because most of the comments are located in the upper levels. The post falls 4, 412 positions from a ranking based only on the number of comments to its rank based on the h-index.

Figure 10c shows a histogram with the number of posts with a given h-index. This distribution is less skewed than those of the number of comments and the maximum depth (compare with Figures 10a and 10b). In Figures 13 and 14 we plot a $3 \mathrm{D}$ chart to compare both, number of comments and maximum depth, against the h-index.

Although we observe an evident correlation between both quantities (more pronounced in the maximum depth) there exist posts which receive a lot of comments but interestingly do not have a significant h-index. This is even more evident when comparing h-index and maximum depth.

Since many posts share the same h-index, we need a way to break the ties. In this situation, we prioritize posts which reach a certain h-index with less comments. Thus, our final proposed measure uses as a first ranking criteria the h-index and as second the inverse of the number of comments. For a post $i$ we use the following formula to rank it:

$$
r_{i}=\text { h-index } i+\frac{1}{\text { num comments }_{i}} .
$$

The first 15 posts according to this ranking can be seen in Table 4. We also show their ranks if only their number of comments or their maximal discussion depth would have been considered. In the latter case, we choose as well the number of comments to break the ties.

\section{CONCLUSIONS}

Our analysis represents a step toward the understanding of the structure of networks in which relations are hidden and more generic than explicit, well-defined links such as friendships or affiliations. The Slashdot network exhibits some special features that deviate from traditional social networks: neutral mixing by degree, almost identical in and out degree distributions, only moderated reciprocity, and absence of a complex community structure. We conjecture that most of the reactions in Slashdot arise when high diversity in opinions occur. Users are therefore more inclined to be linked to people who express different points of view [17]. The nature of this interaction seems to be a key aspect to understand the obtained results.

Unlike the BBS network [24, 8] where discussions are unrestricted, the scoring system of Slashdot guarantees a high quality and representativity of the social interaction. This particular feature allowed us to find a correlation between scores and number of received replies and to distinguish clearly between two classes of users: good writers who, on average achieve high scores for their comments, and regular writers. The number of replies of a comment depends mostly on its quality (the score it achieved) but we find some weak evidence for user reputation influencing the connectivity in the network. Good writers are more likely than regular ones to receive replies to occasional comments with low scores. However, this effect is not strong enough to cause assortative mixing by score since the opposite is not true. Regular writers can expect a similar number of replies as good writers to their comments with high scores, so there is no negative effect of a user's reputation.

When analyzing the tree structure generated by the nesting of comments, we find interesting properties such as selfsimilarity within the different nesting levels of a discussion. This suggests that, despite the strong heterogeneity in the shapes of the discussions visible in their radial tree representation, a simple depth-invariant mechanism exists which is responsible for their evolution. A detailed study of the dynamics governing the growth of nested discussions is a topic of ongoing research.

To measure the degree of controversy of a discussion, a recent approach [15] trains a classifier using features that combine semantic and structural information. Our proposed measure, based on the h-index, appears to be a more convenient indicator because of its simplicity, objectivity and robustness. It can be calculated efficiently and is monotonic (it never decreases), which makes it also a stable quantity to monitor and rank a discussion thread while it is still alive and receiving contributions. However, human based visual validation is necessary to check how it correlates with subjective sensation of controversy. 


\begin{tabular}{|c|c|cc|cc|l|}
\hline$\#$ & H & \multicolumn{2}{|c|}{ Num cmnts (\#) } & \multicolumn{2}{|c|}{ Depth (\#) } & Title \\
\hline 1 & 11 & 527 & $(401)$ & 16 & $(113)$ & Violating A Patent As Moral Choice \\
2 & 11 & 529 & $(390)$ & 12 & $(1374)$ & Human Genes Still Evolving \\
3 & 11 & 605 & $(208)$ & 16 & $(120)$ & Powell Aide Says Case for War a 'Hoax' \\
4 & 11 & 693 & $(96)$ & 17 & $(34)$ & US Releasing 9/11 Flight 77 Pentagon Crash Tape \\
5 & 10 & 243 & $(3287)$ & 15 & $(159)$ & Apple Fires Five Employees for Downloading Leopard \\
6 & 10 & 288 & $(2431)$ & 14 & $(356)$ & Linus Speaks Out On GPLv3 \\
7 & 10 & 290 & $(2409)$ & 11 & $(1774)$ & New Mammal Species Found in Borneo \\
8 & 10 & 309 & $(2078)$ & 13 & $(698)$ & Biofuel Production to Cause Water Shortages? \\
9 & 10 & 315 & $(1999)$ & 12 & $(1168)$ & Torvalds on the Microkernel Debate \\
10 & 10 & 355 & $(1511)$ & 17 & $(17)$ & Well I'll Be A Monkey's Uncle \\
11 & 10 & 361 & $(1446)$ & 13 & $(747)$ & Windows Vista Delayed Again \\
12 & 10 & 366 & $(1394)$ & 14 & $(416)$ & NSA Had Domestic Call Monitoring Before 9/11? \\
13 & 10 & 367 & $(1379)$ & 11 & $(1922)$ & Unleashing the Power of the Cell Broadband Engine \\
14 & 10 & 380 & $(1279)$ & 12 & $(1238)$ & Making Ice Without Electricity \\
15 & 10 & 384 & $(1243)$ & 14 & $(424)$ & Evidence of the Missing Link Found? \\
\hline
\end{tabular}

Table 4: Top-15 controversial posts according to our proposed measure and corresponding positions according to the number of comments and maximum depth rankings.

\section{ACKNOWLEDGMENTS}

We thank the reviewers for their valuable suggestions and Càtedra Telefònica de Producció Multimèdia for funding.

\section{REFERENCES}

[1] A. Barrat, M. Barthélemy, R. Pastor-Satorras, and A. Vespignani. The architecture of complex weighted networks. Proc. Natl. Acad. Sci. USA, 101(11):3747-3752, March 2004.

[2] L. Bornmann and H. D. Daniel. What do we know about the h index? J. Am. Soc. Inf. Sci. Tech., 58:1381-1385, 2007.

[3] T. Braun, W. Glanzel, and A. Schubert. A Hirsch-type index for journals. Scientist, 19:8-8, 2005.

[4] A. B. Brush, X. Wang, T. C. Turner, and M. A. Smith. Assessing differential usage of usenet social accounting meta-data. In Proc. SIGCHI '05, pages 889-898, New York, NY, USA, 2005. ACM.

[5] A. Clauset, C. R. Shalizi, and M. E. J. Newman. Power-law distributions in empirical data. arXiv:0706.1062, June 2007.

[6] D. Fisher, M. Smith, and H. T. Welser. You are who you talk to: Detecting roles in usenet newsgroups. In Proc. HICSS '06, Washington, USA, 2006. IEEE CS.

[7] D. Garlaschelli and M. I. Loffredo. Patterns of link reciprocity in directed networks. Phys. Rev. Lett, 93(26):268701, 2004.

[8] K.-I. Goh, Y.-H. Eom, H. Jeong, B. Kahng, and D. Kim. Structure and evolution of online social relationships: Heterogeneity in unrestricted discussions. Phys. Rev. E, 73(6):066123, 2006.

[9] J. E. Hirsch. An index to quantify an individual's scientific research output. Proc. Natl. Acad. Sci. USA, 102(46):16569-16572, 2005.

[10] P. Holme, C. R. Edling, and F. Liljeros. Structure and time evolution of an internet dating community. Social Networks, 26(2):155-174, 2004.

[11] A. Kaltenbrunner, V. Gómez, and V. López. Description and prediction of Slashdot activity. In Proc. 5th Latin American Web Congress (LA-WEB 2007), Santiago de Chile, 2007. IEEE CS.
[12] A. Kaltenbrunner, V. Gómez, A. Moghnieh, R. Meza, J. Blat, and V. López. Homogeneous temporal activity patterns in a large online communication space. In Proc. BIS 2007 Workshop on Social Aspects of the Web (SAW 2007). Poland, 2007.

[13] C. Lampe and P. Resnick. Slash(dot) and burn: Distributed moderation in a large online conversation space. In Proc. SIGCHI '04, pages 543-550, New York, USA, 2004. ACM.

[14] N. Matsumura, D. Goldberg, and X. Llora. Mining directed social network from message board. In Proc. 14th $W W W$ '05, pages 1092-1093, New York, USA. ACM Press., 2005.

[15] G. Mishne and N. Glance. Leave a reply: An analysis of weblog comments. In Third Annual Workshop on the Weblogging Ecosystem: Aggregation, Analysis and Dynamics (WWW'06), Edumburg, UK, May 2006.

[16] M. Mitzenmacher. A brief history of generative models for power law and lognormal distributions. Internet Mathematics, 1(2):226-251, 2003.

[17] R. Munroe, 2008. http://xkcd.com/386/.

[18] M. E. J. Newman. The structure and function of complex networks. SIAM Review, 45(2):167-256, 2003.

[19] M. E. J. Newman and J. Park. Why social networks are different from other types of networks. Phys. Rev. E, 68(3):036122, 2003.

[20] W. Sack. Discourse diagrams: Interface design for very large-scale conversations. In Proc. HICSS '00. Volume 3, page 3034, Washington, DC, USA, 2000. IEEE CS.

[21] A. Sidiropoulos and Y. Manolopoulos. Generalized comparison of graph-based ranking algorithms for publications and authors. Journal of Systems and Software, 79(12):1679-1700, 2006.

[22] M. Smith. Tools for navigating large social cyberspaces. Commun. ACM, 45(4):51-55, 2002.

[23] D. J. Watts and S. H. Strogatz. Collective dynamics of small-world networks. Nature, 393(6684):440-442, 1998.

[24] K. Zhongbao and Z. Changshui. Reply networks on a bulletin board system. Phys. Rev. E, 67(3):036117, 2003. 